coherent transfer determines the amount of decoherence; the basis in which the qubit is dephased can be controlled using qubit manipulations performed before and after the procedure. This engineered environment decoheres each qubit independently, but with the same strength.

The team used quantum process tomography to reconstruct the full density matrix of the system for different values of applied decoherence. They tested the density matrix for $1: 3$ and 2:2 entanglement, for distillability, for violation of a Bell-like inequality and for its usefulness for 'entanglement superactivation' - a multi-party protocol similar to distillation ${ }^{3}$. From this information, they divided up the state evolution during decoherence into regions of different properties. The progression is shown in Fig. 1. As the initial entangled state is decohered, they first observe that the state stops violating a Bell inequality. With increasing decoherence the state is no longer suitable for super-activation. At one further point, they see that the entanglement and distillability with respect to all possible 2:2 partitions disappear but those with respect to any of the $1: 3$ partitions are still present - this is the signature of bound entanglement. As more decoherence is introduced, the 1:3 entanglement also disappears, leaving a fully separable state.

Barreiro et al. harness state-of-the-art control for generation and manipulation of entangled states, and complement this with a rigorous theoretical analysis. The controlled progression of multipartite entanglement is made possible by the admirable precision achieved in the experiments, both in the state initialization and the engineered decoherence. The study not only provides an intriguing view of multipartite entanglement, but also shows the value of engineered decoherence both for controlling quantum systems and as a tool to understand more about quantum states. Engineering different decay channels should make it possible to observe a variety of entanglement progressions, which will give us further insights into the nature of decoherence and entanglement ${ }^{4}$. Extending these techniques could also provide novel methods for quantum state and process engineering ${ }^{5,6}$, opening a new playground for quantum science.

Jonathan Home is in the Institute for Quantum Electronics, ETH Zurich, Hoenggerberg, Zurich 8093, Switzerland.

e-mail: jhome@phys.ethz.ch

References

1. Barreiro, J. T. et al. Nature Phys. 6, 943-946 (2010).

2. Horodecki, M., Horodecki, P. \& Horodecki, R. Phys. Rev. Lett. 80, 5239-5242 (1998)

3. Shor, P. W., Smolin, J. A. \& Thapliyal, A. V. Phys. Rev. Lett. 90, 107901 (2003)

4. Aolita, L. et al. Phys. Rev. Lett. 100, 080501 (2008)

5. Diehl, S. et al. Nature Phys. 4, 878-883 (2008).

6. Verstraete, F., Wolf, M. M. \& Cirac, J. I. Nature Phys. 5, 633-636 (2009).

\title{
HISTORY OF PHYSICS
}

\section{The mighty ocean}

For physics, the first half of the twentieth century was a time of profound transformation, bringing about the transition from what we now call 'classical' to 'modern' physics. With the new concepts and fields of study there emerged a global physics community, an ever-growing network of collaboration and scientific exchange. Yves Gingras has analysed hundreds of thousands of scientific papers published between 1900 and 1945, and identified several trends that characterize this transformative period (Phys. Perspect. $12,248-265 ; 2010$ ).

In the early years of the twentieth century, the sensation of there being a mountain to climb in exploring and understanding the emerging concepts of quantum mechanics and relativity (among others) must have been overwhelming. That such a challenge can be met only through collaborative effort is beautifully captured in the words of Paul Drude, who delivered his inaugural address as a member of the Prussian Academy of Sciences on 28 June 1906 (and committed suicide only one week later): "In these times of rapid progress, when for each physicist there are plenty of tasks on offer, worries also arise that the skill and capacity of an individual permits the achievement of merely a small fraction of

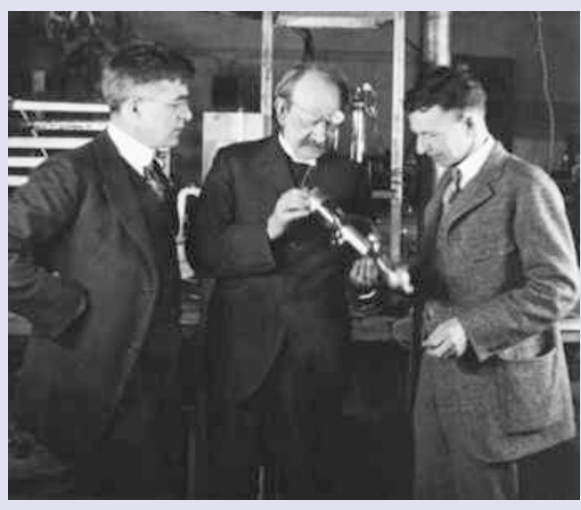

right). Thomson maintained his centre position until the mid-1920s, when others, including Arnold Sommerfeld, Werner Heisenberg, Arthur Compton and Hans Bethe, started to take over.

These changes reflect a shift in 'mainstream' topics, from electron and atomic physics, to quantum mechanics, to nuclear and solid-state physics. Indeed, no single physicist remained in a central position in the co-citation network for more than 15 or 20 years.

But the first half of the last century was also a time of radical political upheaval. Among the consequences were a decrease of German influence in physics and rapid growth in American physics research after the 1920s, as Gingras's detailed data show. On top of these changes, his study reveals a steady increase in the number of physicists and the subdivision of physics into ever more subfields, together with a trend towards publications with several authors, rather than single-author papers.

"Little drops of water, / Little grains of sand, / Make the mighty ocean / And the pleasant land", says the poem commonly attributed to the American Julia Fletcher Carney. It holds some truth for physics as well.

ANDREAS TRABESINGER 